\title{
STEADY-STATE COMPUTATIONS OF GRANULAR FLOW IN AN AXISYMMETRIC HOPPER*
}

\author{
FENG WANG $\dagger$, CARL L. GARDNER $\ddagger$, AND DAVID G. SCHAEFFER
}

\begin{abstract}
Axisymmetric steady-state computations of plastic incompressible granular flow in a converging hopper are presented. The granular flow equations are solved as a first-order system of conservation laws plus a set of constitutive relations, using a conservative finite difference method. Newton's method is used to solve the nonlinear partial differential equations (PDEs).

The steady-state granular flow equations may change type from elliptic to hyperbolic. In this investigation, flow parameters and boundary conditions are chosen to ensure that the PDE system is elliptic. The transition from a "radial solution" similarity flow at the top of the hopper to a more general flow involving a boundary layer near the exit of the hopper is analyzed.
\end{abstract}

Key words. granular flow, nonlinear partial differential equations (PDEs), conservation laws, finite difference methods, Newton's method

AMS(MOS) subject classifications. 76A10, 76M20

1. Introduction. Many challenging scientific problems are posed by the flow of granular materials in a hopper. Regardless of the geometry, the governing partial differential equations (PDEs) (in a continuum formulation) are vulnerable to change of type [5], often leading to ill-posed initial value problems. In the silo/hopper geometry, the flow is often complicated by the presence of free boundaries separating regions undergoing plastic deformation from stagnant, or at least nondeforming, regions. Not only is it difficult to include such boundaries in a computation, but the governing equations become singular as the deformation rate tends to zero. Moreover, the modeler's task is complicated by the bewildering variety of constitutive laws that have been proposed in the literature.

As an initial investigation in this daunting environment, we make the following simplifying assumptions. (i) We use the conceptually simplest constitutive law due essentially to Coulomb [3]. (ii) We choose the geometry to eliminate the risk of equations that change type. (iii) We solve a problem on a fixed domain, disregarding a free boundary at the hopper exit (and the free-boundary condition). (iv) We solve steady-state equations, neglecting the very important possibility of time-dependent flow. (v) We consider only axisymmetric flow, not fully three-dimensional flow. Even with all these simplifications, the unusual structure of the granular flow equations presents challenging problems, and these are the subjects of this paper.

2. Granular flow equations. The plastic flow of an incompressible granular material is described by conservation laws for mass and momentum, with a constitutive law relating stress and strain. The constitutive law (3), the simplest of many proposed,

* Received by the editors August 12, 1991; accepted for publication August 28, 1991.

$\dagger$ Department of Applied Mathematics and Statistics, University at Stony Brook, Stony Brook, New York 11794.

\$Department of Computer Science, Duke University, Durham, North Carolina 27706. This author's research was supported in part by National Science Foundation grant DMS-8804592, which includes funds from the Air Force Office of Scientific Research.

$\S$ Department of Mathematics, Duke University, Durham, North Carolina 27706. This author's research was supported in part by National Science Foundation grant DMS-8804592, which includes funds from the Air Force Office of Scientific Research. 
assumes incompressibility and rigid perfectly plastic behavior. (See [5] for a mathematical derivation.) The granular flow equations are

$$
\begin{gathered}
\nabla \cdot \mathbf{v}=0, \\
\rho \frac{\partial v_{i}}{\partial t}=-\frac{\partial T_{i j}}{\partial x_{j}}+\rho g_{i}, \\
T_{i j}=\sigma \delta_{i j}+k \sigma \frac{V_{i j}}{|V|},
\end{gathered}
$$

where $\mathbf{v}$ is the velocity, $\rho$ is the density, $T$ is the stress tensor (with compressive stress positive), $\mathbf{g}=-g \hat{\mathbf{z}}$ is the gravitational acceleration, $\sigma=$ Trace $\{T\} / 3$ is the average or normal stress, $k \in(0, \sqrt{3 / 2}]$ is a dimensionless material constant specifying the angle of internal friction, $V$ is the strain rate or deformation rate tensor

$$
V_{i j}=-\frac{1}{2}\left(\frac{\partial v_{i}}{\partial x_{j}}+\frac{\partial v_{j}}{\partial x_{i}}\right)
$$

and $|V|$ is the Euclidean norm of $V$. Repeated spatial indices $i, j=1,2,3$ are summed over. Equation (1) expresses conservation of mass, (2) expresses conservation of momentum, and (3) is the granular analogue of the von Mises constitutive law for plastic deformation of metals. In (2) we have dropped the advective term $\rho \mathbf{v} \cdot \nabla v_{i}$, since, for the slow flows in typical industrial hoppers, this term is much smaller than the other terms in (2).

We numerically solve for the steady-state solution of (1)-(3) in an axisymmetric converging hopper in spherical coordinates

$$
\left\{(r, \theta, \varphi) \mid R_{\text {inner }} \leqq r \leqq R_{\text {outer }}, 0 \leqq \theta \leqq \theta_{w}, 0 \leqq \varphi<2 \pi\right\},
$$

where $R_{\text {inner }}$ and $R_{\text {outer }}$ are, respectively, the inner and outer computational boundaries of the hopper, and $\theta_{w}$ is the angle between the axis and the conical wall of the hopper (see Fig. 1). The steady-state flow is specified by the geometrical parameters $R_{\text {outer }} / R_{\text {inner }}$ and $\theta_{w}$, and the material parameters $k$ and wall friction $\delta_{w}$ (set equal to zero for the present study). We measure stresses in terms of $\rho g R_{\text {inner }}$. Note that the velocity can be scaled by an overall factor.

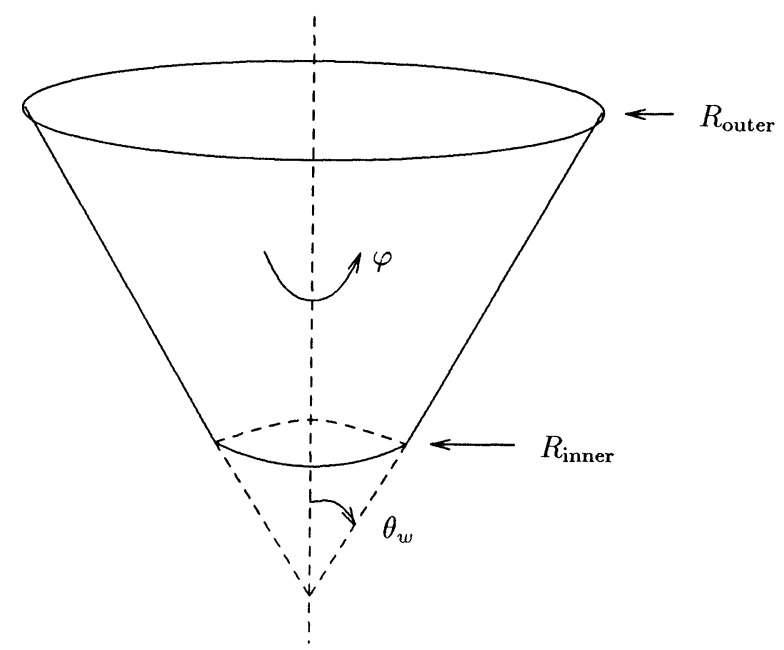

FIG. 1. Schematic of an axisymmetric industrial hopper. 
Imposing axial symmetry implies that

$$
\frac{\partial}{\partial \varphi}=0, \quad v_{\varphi}=0
$$

and that $T$ has the form

$$
T=\left(\begin{array}{ccc}
T_{r r} & T_{r \theta} & 0 \\
T_{r \theta} & T_{\theta \theta} & 0 \\
0 & 0 & T_{\varphi \varphi}
\end{array}\right)
$$

In steady state, (1)-(3) simplify to the following seven equations in the seven unknowns $v_{r}, v_{\theta}, \sigma, T_{r r}, T_{\theta \theta}, T_{\varphi \varphi}$, and $T_{r \theta}$ :

$$
f_{m}=\partial_{r}\left(r^{2} \sin \theta v_{r}\right)+\frac{1}{r} \partial_{\theta}\left(r^{2} \sin \theta v_{\theta}\right)=0
$$

(9) $f_{r}=\partial_{r}\left(r^{2} \sin \theta T_{r r}\right)+\frac{1}{r} \partial_{\theta}\left(r^{2} \sin \theta T_{r \theta}\right)-r \sin \theta\left(T_{\theta \theta}+T_{\varphi \varphi}\right)+\left(r^{2} \sin \theta\right) \rho g \cos \theta=0$,

$$
\begin{gathered}
f_{\theta}=\partial_{r}\left(r^{2} \sin \theta T_{r \theta}\right)+\frac{1}{r} \partial_{\theta}\left(r^{2} \sin \theta T_{\theta \theta}\right)+r \sin \theta\left(T_{r \theta}-T_{\varphi \varphi} \cot \theta\right) \\
-\left(r^{2} \sin \theta\right) \rho g \sin \theta=0, \\
f_{r r}=|V|\left(T_{r r}-\sigma\right)+k \sigma \partial_{r} v_{r}=0, \\
f_{\theta \theta}=|V|\left(T_{\theta \theta}-\sigma\right)+\frac{k \sigma}{r}\left(\partial_{\theta} v_{\theta}+v_{r}\right)=0, \\
f_{\varphi \varphi}=|V|\left(T_{\varphi \varphi}-\sigma\right)+\frac{k \sigma}{r}\left(v_{\theta} \cot \theta+v_{r}\right)=0, \\
f_{r \theta}=|V| T_{r \theta}+\frac{k \sigma}{2}\left(\partial_{r} v_{\theta}+\frac{1}{r}\left(\partial_{\theta} v_{r}-v_{\theta}\right)\right)=0 .
\end{gathered}
$$

Equation (8) is the mass conservation law, (9) and (10) are the momentum conservation laws, and (11)-(14) are the constitutive relations. We multiplied (1) and (2) by the Jacobian $r^{2} \sin \theta$ of the transformation from Cartesian to spherical coordinates to put the continuity and momentum equations in conservation form in spherical coordinates.

The condition $\sigma=\operatorname{Trace}\{T\} / 3$ is enforced by the sum of (11)-(13) plus the continuity equation (8).

For axisymmetric problems, the strain rate tensor $V$ has the following components in spherical coordinates:

$$
\begin{gathered}
V_{r r}=-\partial_{r} v_{r}, \quad V_{\theta \theta}=-\frac{1}{r}\left(\partial_{\theta} v_{\theta}+v_{r}\right), \quad V_{\varphi \varphi}=-\frac{1}{r}\left(v_{\theta} \cot \theta+v_{r}\right), \\
V_{r \theta}=-\frac{1}{2}\left(\partial_{r} v_{\theta}+\frac{1}{r}\left(\partial_{\theta} v_{r}-v_{\theta}\right)\right), \quad V_{r \varphi}=0, \quad V_{\theta \varphi}=0,
\end{gathered}
$$

where $|V|$ is given by

$$
|V|^{2}=V_{r r}^{2}+V_{\theta \theta}^{2}+V_{\varphi \varphi}^{2}+2 V_{r \theta}^{2} .
$$

Note that, for axisymmetric flow, $V_{\varphi \varphi}$ does not contain any velocity derivatives.

For an infinite hopper, there is a similarity solution of the steady-state equations

$$
v_{r}=-\frac{u(\theta)}{r^{2}}, \quad v_{\theta}=0, \quad T_{i j}=r T_{i j}(\theta),
$$

with $u(\theta)>0$ and $T_{\theta \theta}=T_{\varphi \varphi}$. This "radial solution" was discovered by Jenike [4]. $u(\theta)$ 
is the solution of an ordinary differential equation. We analyze the transition from a radial solution flow at the top of the hopper to a more general flow near the exit of the hopper.

The principal part of the granular flow equations (1)-(3) is sixth-order and can have both elliptic and hyperbolic modes in steady state. For a sufficiently steep smooth hopper, the principal part of the steady-state granular flow equations is elliptic [5]. In this investigation we focus on elliptic flow in the hopper. Therefore, we must specify three boundary conditions at the top and bottom computational boundaries and along the hopper wall. Since $v_{\varphi}=0$, we must specify two additional boundary conditions.

At the axis of the hopper, we impose the symmetry conditions: $v_{\theta}$ and $T_{r \theta}$ are odd functions of $\theta$, while $v_{r}, \sigma, T_{r r}, T_{\theta \theta}$, and $T_{\varphi \varphi}$ are even functions of $\theta$.

At the top "inflow" computational boundary $r=R_{\text {outer }}$, we specify $v_{r}$ and $v_{\theta}$ from the radial solution. ${ }^{1}$ At the bottom "outflow" computational boundary $r=R_{\text {inner }}$, we specify that $V_{r \theta}=0$ and $v_{r}=v_{r}(\theta)$. Along the hopper wall, $v_{\theta}=0$. Assuming the wall is frictionless, we also specify that $T_{r \theta}=0$ along the wall.

The current investigation may be viewed as a prelude to computing the free outflow surface that marks the boundary between converging flow in the hopper and rigid "plug" flow with no deformation below the hopper. With a free outflow surface, we would prescribe $v_{\theta}$ in addition to $v_{r}$ and $V_{r \theta}$. Rigid plug flow below the hopper may be achieved experimentally by connecting a cylindrical tube with a piston to the exit of the hopper and withdrawing the piston with a uniform velocity. The experiments of [2] implemented this type of boundary condition in a different configuration.

We now show that if we specify vertical flow at the outflow boundary, then the problem becomes hyperbolic near the outflow boundary. In this investigation, we restrict our attention to elliptic flows.

To prove that the problem becomes hyperbolic near the outflow boundary for

$$
v_{r}=-v \cos \theta, \quad v_{\theta}=v \sin \theta, \quad v_{\varphi}=0,
$$

we first combine (15) and (18) to conclude that $V_{\varphi \varphi}=0$. Since the material is incompressible, contraction along one direction must be accompanied by expansion along another direction, i.e., Trace $\{V\}=\nabla \cdot \mathbf{v}=0$.

The strain rate tensor has the following form for axisymmetric problems:

$$
V=\left(\begin{array}{ccc}
V_{r r} & V_{r \theta} & 0 \\
V_{r \theta} & V_{\theta \theta} & 0 \\
0 & 0 & V_{\varphi \varphi}
\end{array}\right) .
$$

The trace of the upper left $2 \times 2$ block is zero since Trace $\{V\}=0$ and $V_{\varphi \varphi}=0$. Since $V \neq 0$, the two eigenvalues of the upper left $2 \times 2$ block must be equal in magnitude and opposite in sign. The middle eigenvalue of $V$ is zero. We have proved the following lemma.

LeмmA 1. At a point where the velocity is vertical, the middle eigenvalue of the strain rate tensor $V$ is zero.

Using the stability analysis results in [5], we prove the following proposition.

Proposition 1. The steady-state granular flow equations (8)-(14) are hyperbolic if the flow is vertical.

Proof. Section 3 of [5] shows that the type of the granular flow equations depends on the middle eigenvalue $a_{2}$ of $|V|^{-1} V$. Specifically, $a_{2}=0$ implies that the steady-state

\footnotetext{
${ }^{1}$ If "far-field" boundary conditions for all seven variables are specified at $r=R_{\text {outer }}$ from the radial solution, then the outer radius $R_{\text {outer }}$ must be $\gtrsim 10 R_{\text {inner }}$ to prevent small oscillations from occurring in $v_{\theta}$ and $T_{r \theta}$ near $R_{\text {outer }}$.
} 
equations are of hyperbolic type. $a_{2}=0$ follows if the velocity is vertical since Lemma 1 remains true for $|V|^{-1} V$ as long as $V \neq 0$.

3. Numerical method. The granular flow equations (8)-(14) are discretized using the box method (flux method) [1] in spherical coordinates with central differences.

Since the Jacobian of the transformation of coordinates $r^{2} \sin \theta$ vanishes at the axis of the hopper, we impose the conservation laws (8)-(10) at $\theta=\Delta \theta / 2$, instead of at $\theta=0$. We use one-sided first-order accurate derivatives at the wall and the outflow boundary.

We use Newton's method to solve the following discrete granular equations (8)-(14):

$$
J\left[\begin{array}{c}
\delta \sigma \\
\delta v_{r} \\
\delta v_{\theta} \\
\delta T_{r r} \\
\delta T_{\theta \theta} \\
\delta T_{\varphi \varphi} \\
\delta T_{r \theta}
\end{array}\right]=-\left[\begin{array}{c}
f_{m} \\
f_{r} \\
f_{\theta} \\
f_{r r} \\
f_{\theta \theta} \\
f_{\varphi \varphi} \\
f_{r \theta}
\end{array}\right]=-f,
$$

where $J$ is the Jacobian and $f$ is the $7 \times 1$ residual. Note that $f_{m}, f_{r}$, and $f_{\theta}$ are linear. The new solution is obtained by setting

$$
\left[\begin{array}{c}
\sigma \\
v_{r} \\
v_{\theta} \\
T_{r r} \\
T_{\theta \theta} \\
T_{\varphi \varphi} \\
T_{r \theta}
\end{array}\right] \leftarrow\left[\begin{array}{c}
\sigma \\
v_{r} \\
v_{\theta} \\
T_{r r} \\
T_{\theta \theta} \\
T_{\varphi \varphi} \\
T_{r \theta}
\end{array}\right]+\left[\begin{array}{c}
\delta \sigma \\
\delta v_{r} \\
\delta v_{\theta} \\
\delta T_{r r} \\
\delta T_{\theta \theta} \\
\delta T_{\varphi \varphi} \\
\delta T_{r \theta}
\end{array}\right]
$$

The discretized Jacobian has the following block structure:

$$
J=\left[\begin{array}{ccccccc}
0 & \frac{\delta f_{m}}{\delta v_{r}} & \frac{\delta f_{m}}{\delta v_{\theta}} & 0 & 0 & 0 & 0 \\
0 & 0 & 0 & \frac{\delta f_{r}}{\delta T_{r r}} & \frac{\delta f_{r}}{\delta T_{\theta \theta}} & \frac{\delta f_{r}}{\delta T_{\varphi \varphi}} & \frac{\delta f_{r}}{\delta T_{r \theta}} \\
0 & 0 & 0 & 0 & \frac{\delta f_{\theta}}{\delta T_{\theta \theta}} & \frac{\delta f_{\theta}}{\delta T_{\varphi \varphi}} & \frac{\delta f_{\theta}}{\delta T_{r \theta}} \\
\frac{\delta f_{r r}}{\delta \sigma} & \frac{\delta f_{r r}}{\delta v_{r}} & \frac{\delta f_{r r}}{\delta v_{\theta}} & \frac{\delta f_{r r}}{\delta T_{r r}} & 0 & 0 & 0 \\
\frac{\delta f_{\theta \theta}}{\delta \sigma} & \frac{\delta f_{\theta \theta}}{\delta v_{r}} & \frac{\delta f_{\theta \theta}}{\delta v_{\theta}} & 0 & \frac{\delta f_{\theta \theta}}{\delta T_{\theta \theta}} & 0 & 0 \\
\frac{\delta f_{\varphi \varphi}}{\delta \sigma} & \frac{\delta f_{\varphi \varphi}}{\delta v_{r}} & \frac{\delta f_{\varphi \varphi}}{\delta v_{\theta}} & 0 & 0 & \frac{\delta f_{\varphi \varphi}}{\delta T_{\varphi \varphi}} & 0 \\
\frac{\delta f_{r \theta}}{\delta \sigma} & \frac{\delta f_{r \theta}}{\delta v_{r}} & \frac{\delta f_{r \theta}}{\delta v_{\theta}} & 0 & 0 & 0 & \frac{\delta f_{r \theta}}{\delta T_{r \theta}}
\end{array}\right] .
$$

Each block is diagonal, tridiagonal, or tridiagonal with fringes. Newton's equation (20) may be solved directly by banded or sparse matrix techniques with partial pivoting. We use a LINPACK banded solver. 
As an initial guess for the solution, we take the radial solution (17). The Newton method converges quadratically.

4. Computations. We performed numerical simulations of granular flow in a hopper for the following two different outflow boundary conditions:

$$
v_{r}=-u(\theta) / R_{\text {inner }}^{2},
$$

where $u(\theta)$ is determined from the radial solution, and

$$
v_{r}=- \text { const }\left(\varepsilon \cos \left(\pi \theta / \theta_{w}\right)+1\right),
$$

where the constant is determined by requiring the outflowing mass at the bottom of the hopper to be equal to the mass inflowing at the top.

For both boundary conditions (23a) and (23b), we have that $\partial_{\theta} v_{r}=0$ at the corner of the outflow boundary with the wall and with the axis of symmetry since $V_{r \theta}=0$ at the outflow boundary and $v_{\theta}=0$ along the wall and the axis of symmetry (see (15)). This condition ensures that there is no singularity in $v_{r}$ at the corners.

We present computational results for $\theta_{w}=30^{\circ}, \delta=20^{\circ}$, where $k=\sqrt{2} \sin \delta$, $R_{\text {outer }} / R_{\text {inner }}=3$, and boundary conditions (23a) and (23b) at the outflow boundary. All computations were done on an $80 \Delta r \times 20 \Delta \theta$ grid, except for the mesh refinement results in Fig. 11.

In the computations, the overall velocity scale is set (at the inflow boundary) by choosing $u(\theta=0)=1$ in the radial solution (17). The units of stress are chosen so that $\rho g R_{\text {inner }}=1$.

Figures 2-11 illustrate the flow for a hopper with boundary condition (23a). Note that the flow is very close to the radial solution flow. The radial component of the velocity field plotted in Fig. 5 along the outflow surface is just the radial solution $v_{r}$, from boundary condition (23a). The theta component of the velocity field plotted in Figs. 6 and 7 differs from the radial solution in that $v_{\theta} \neq 0$. The average stress $\sigma$ in Fig. 8 is basically linear in $r$ for fixed $\theta$, as in the radial solution (17). Figure 10 shows that $T_{r \theta}$, however, deviates substantially from the radial solution. Both $v_{\theta}$ and $T_{r \theta}$ display an exponentially damped oscillation as functions of $r$ about the radial solution. This type of behavior is also exhibited by solutions of the linearized granular flow equations. Figure 11 indicates convergence of the computations under mesh refinement.

FIG. 2. The velocity field in a hopper for $\theta_{w}=30^{\circ}$ and boundary condition (23a). $80 \times 20$ grid. 


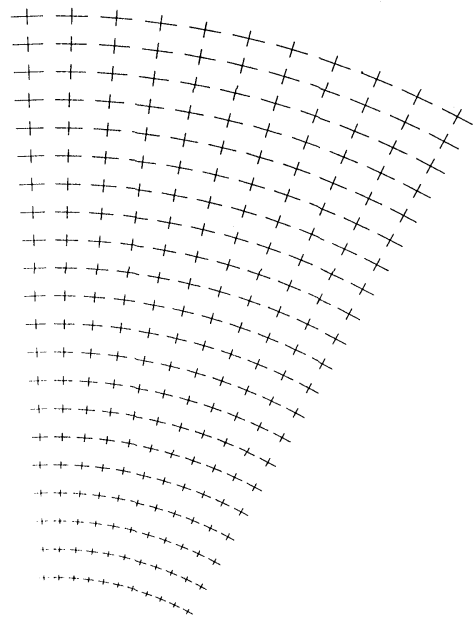

FIG. 3. The eigenvalues and eigendirections of the stress tensor $\left(\theta_{w}=30^{\circ}\right.$, boundary condition (23a)).

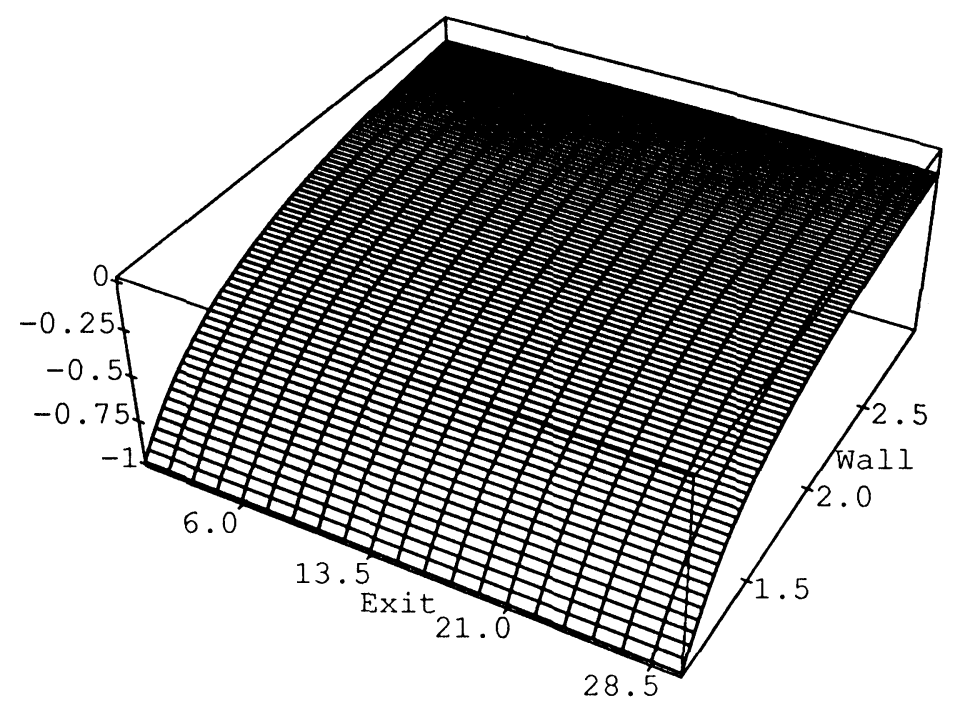

FIG. 4. $v_{r}\left(\theta_{w}=30^{\circ}\right.$, boundary condition (23a)).

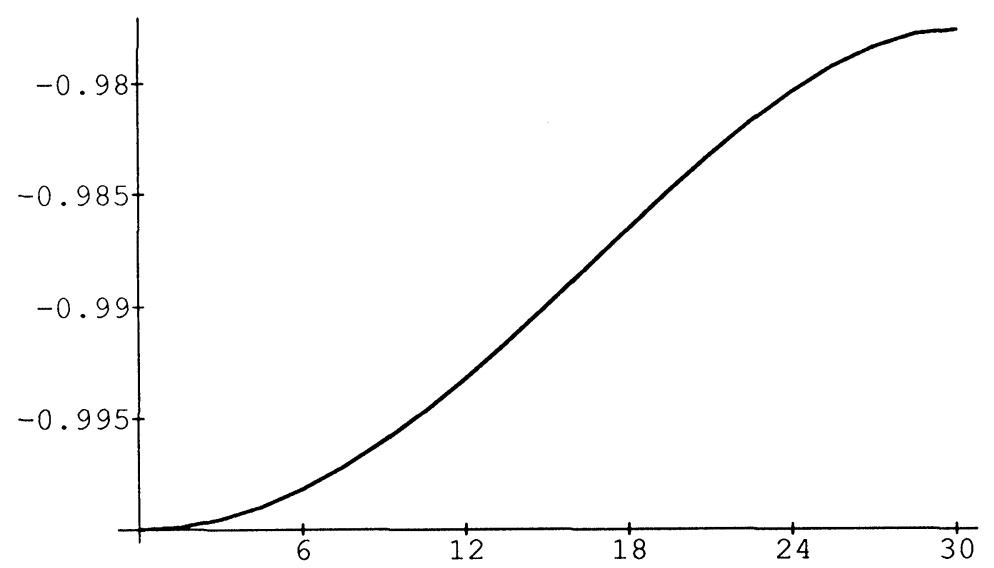

FIG. 5. $v_{r}$ along the outflow surface $\left(\theta_{w}=30^{\circ}\right.$, boundary condition (23a)). 


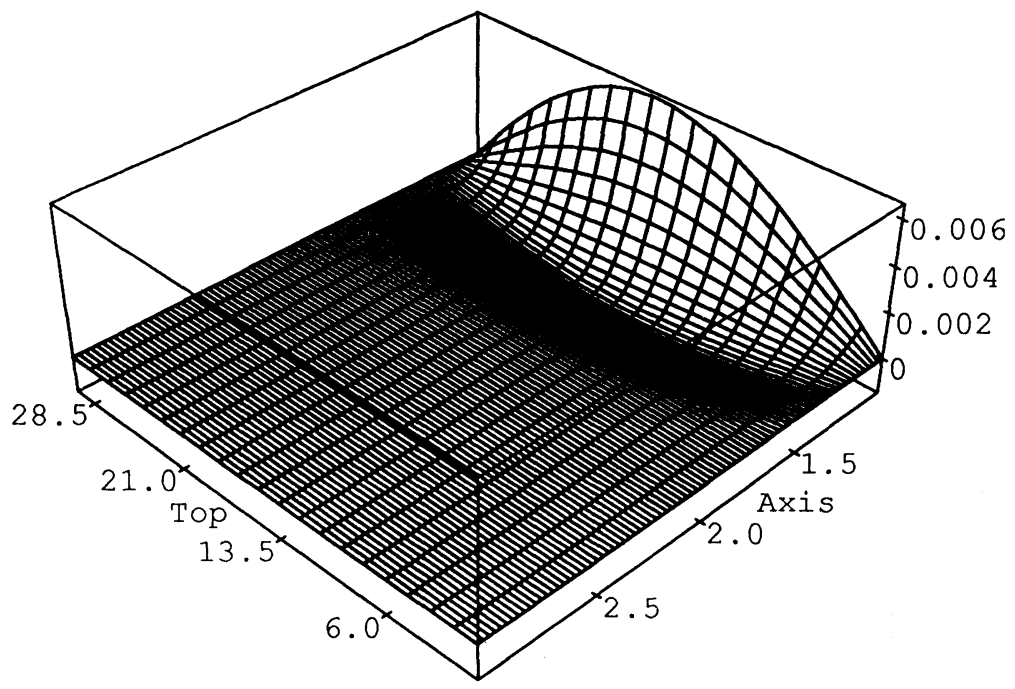

FIG. 6. $v_{\theta}\left(\theta_{w}=30^{\circ}\right.$, boundary condition (23a)).

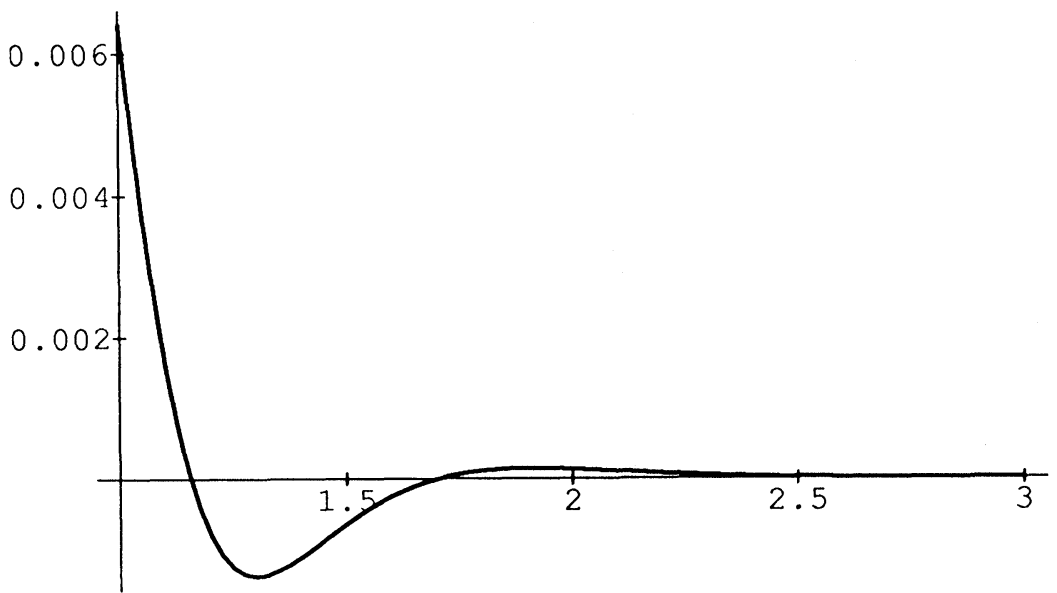

FIG. 7. $v_{\theta}$ along the straight line $\theta=\theta_{w} / 2\left(\theta_{w}=30^{\circ}\right.$, boundary condition (23a)).

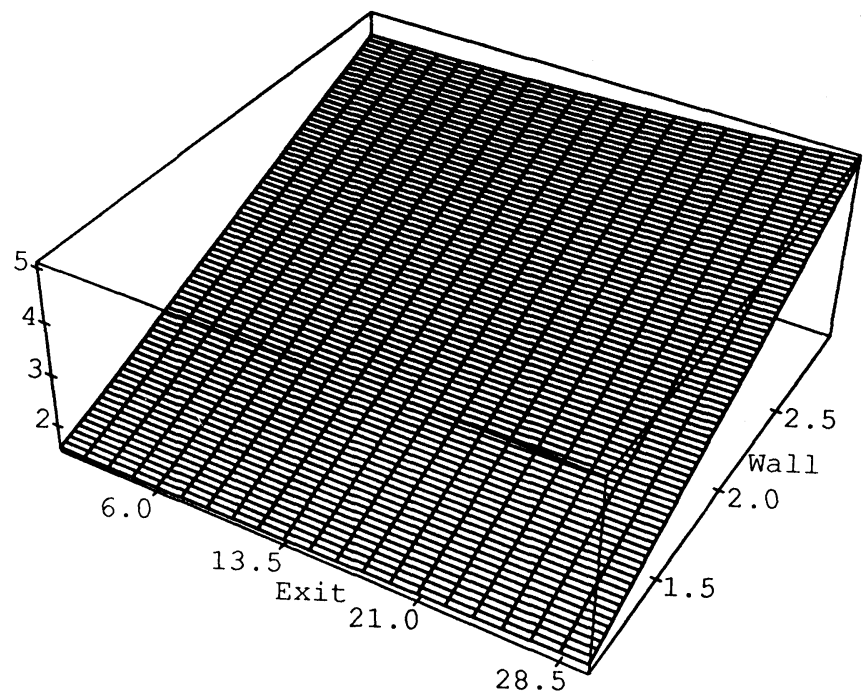

FIG. 8. Average stress $\sigma\left(\theta_{w}=30^{\circ}\right.$, boundary condition (23a)). 


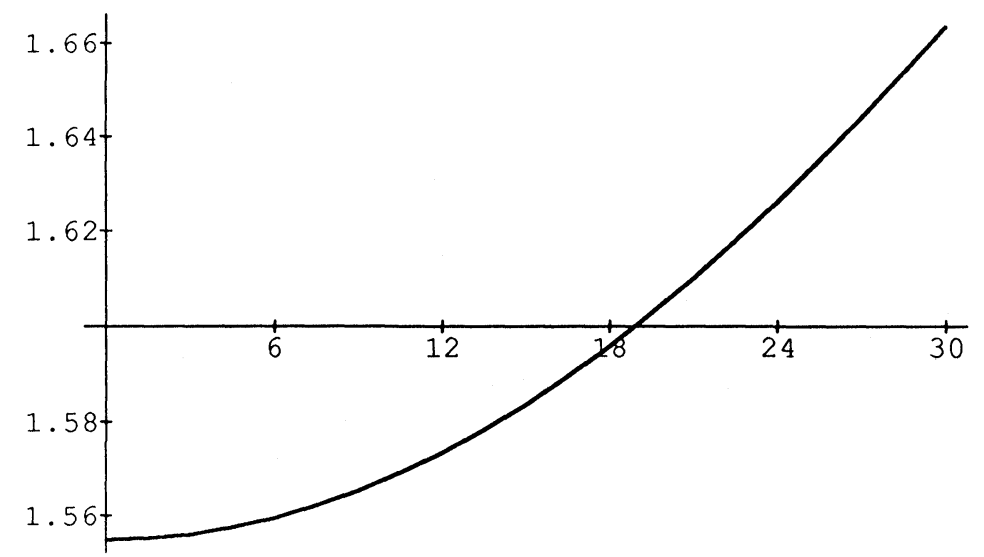

FIG. 9. $\sigma$ along the outflow surface $\left(\theta_{w}=30^{\circ}\right.$, boundary condition (23a)).

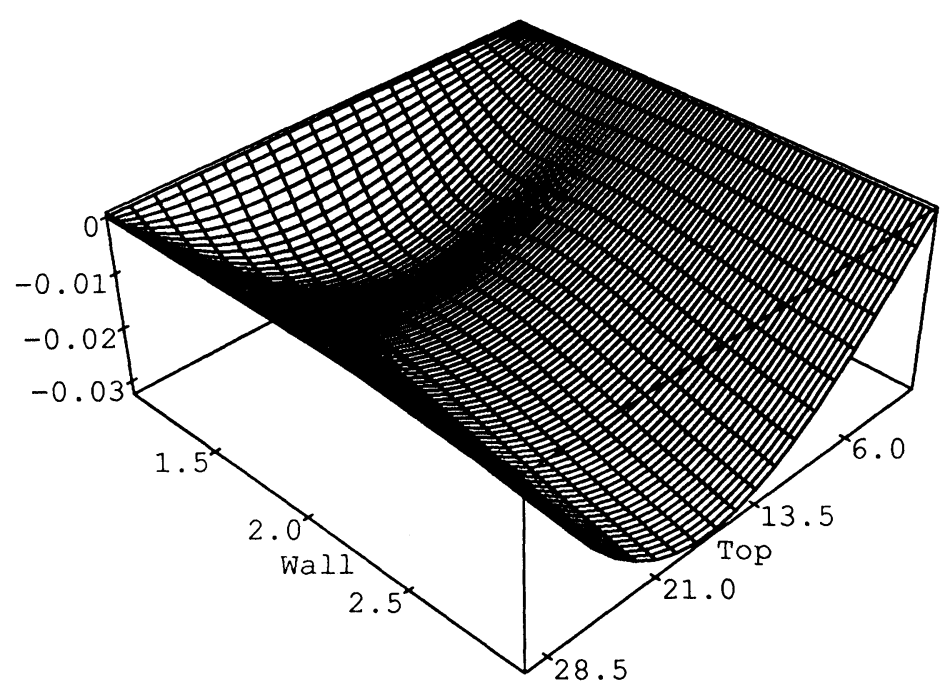

FIG. 10. $T_{r \theta}\left(\theta_{w}=30^{\circ}\right.$, boundary condition (23a)).

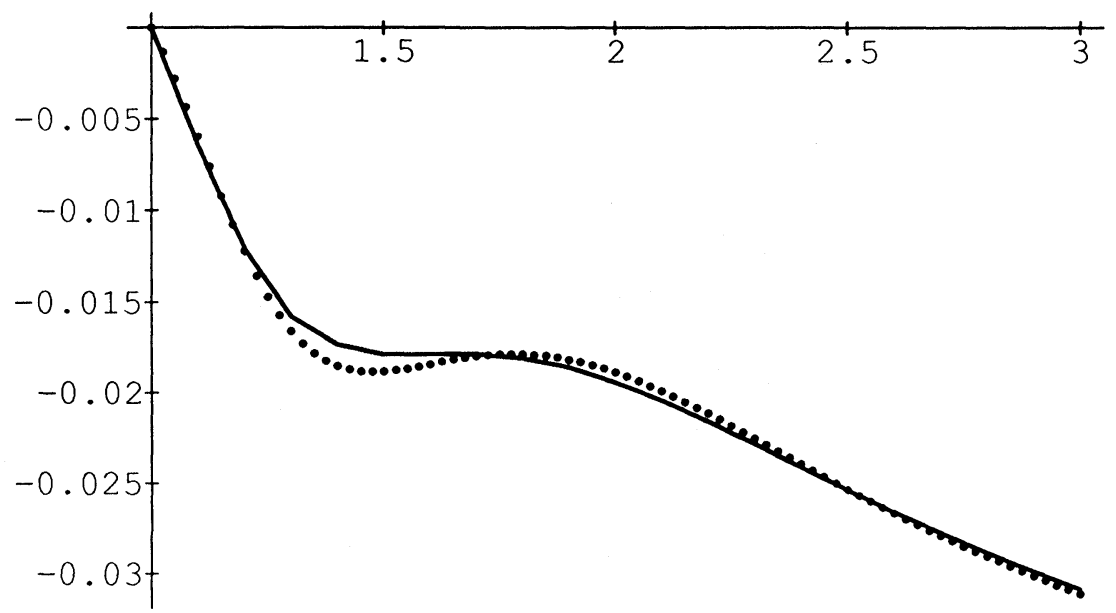

FIG. 11. Convergence under mesh refinement for $T_{r \theta}$ along the straight line $\theta=\theta_{w} / 2\left(\theta_{w}=30^{\circ}\right.$, boundary condition (23a)). $20 \times 20$ (solid) vs. $80 \times 20$ grid (dots). 
Figures 12-17 present a more complicated flow for a hopper with boundary condition (23b). Note the visibly nonradial flow near the corner of the outflow boundary with the wall of the hopper in Figs. 12-14. The average stress $\sigma$ in Fig. 15 is still basically linear in $r$ for fixed $\theta$, except near the corner of the outflow boundary with the wall of the hopper. Again, $v_{\theta} \neq 0$ (see Fig. 16).

The interesting structure in the solutions near the outflow boundary in Figs. 6 and 16 for $v_{\theta}$ and in Figs. 10 and 17 for $T_{r \theta}$ may be understood in the following way. At $r=R_{\text {inner }}, V_{r \theta}=0$. From (15),

$$
V_{r \theta}=-\frac{1}{2 r} \partial_{\theta} v_{r}+\text { terms in } v_{\theta}
$$

Since the first term $\propto \partial_{\theta} v_{r} \neq 0$ from both boundary conditions (23a) and (23b), the second term in (24) is forced to be nonzero at $r=R_{\text {inner }}$. Asymptotically, as $r \rightarrow R_{\text {outer }}$, $v_{\theta} \rightarrow 0$. However, just inside $r=R_{\text {inner }}, v_{\theta}$ remains nonzero, and large gradients develop

FIG. 12. The velocity field for $\theta_{w}=30^{\circ}$ and boundary condition (23b) with $\varepsilon=0.4 .80 \times 20$ grid.

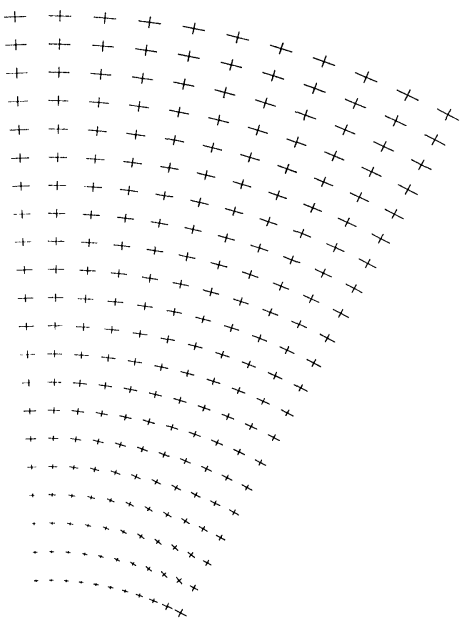

FIG. 13. The eigenvalues and eigendirections of the stress tensor $\left(\theta_{w}=30^{\circ}\right.$, boundary condition (23b) with $\varepsilon=0.4$ ). 


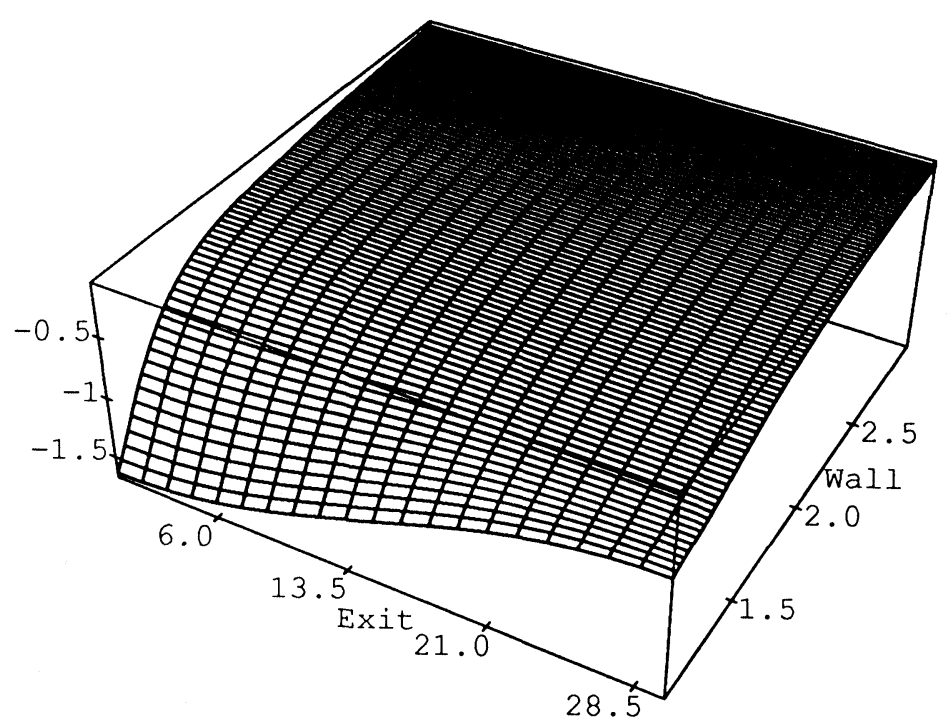

FIG. 14. $v_{r}\left(\theta_{w}=30^{\circ}\right.$, boundary condition (23b) with $\left.\varepsilon=0.4\right)$.

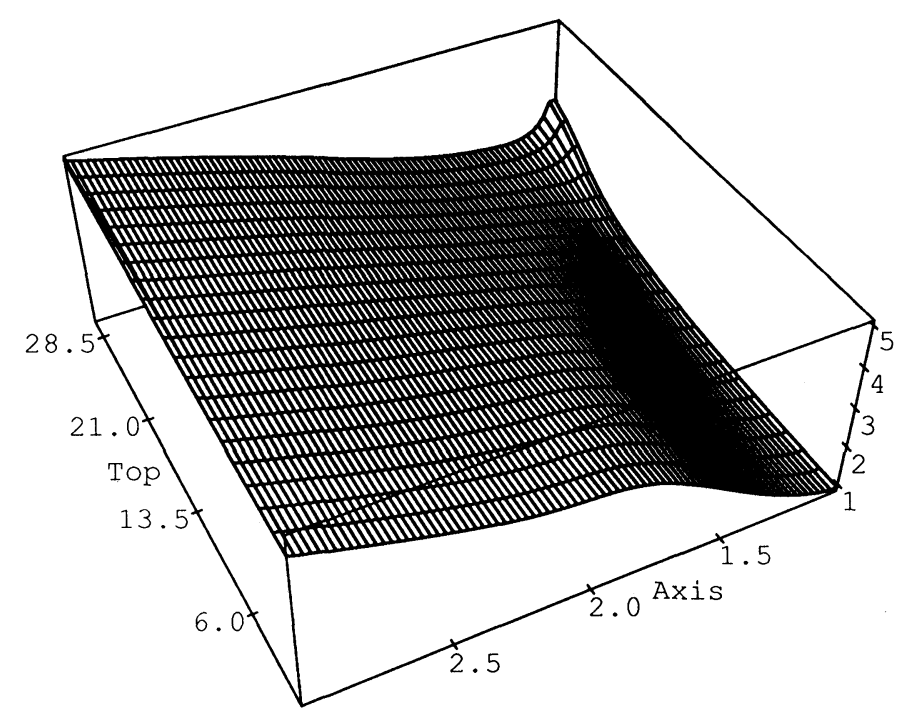

FIG. 15. Average stress $\sigma\left(\theta_{w}=30^{\circ}\right.$, boundary condition (23b) with $\left.\varepsilon=0.4\right)$.

in $v_{\theta}$ and $V_{r \theta}$. Since $T_{r \theta}$ is proportional to $V_{r \theta}$ (see (3)), large gradients develop in $T_{r \theta}$ as well. In this way, a boundary layer in the solutions near $r=R_{\text {inner }}$ is created.

5. Conclusion. The present computations are a "ground-breaking" effort, preparing the way for more difficult problems to follow. These computations study steady flow in a regime where the flow, at least qualitatively, resembles Jenike's radial solution, and are somewhat incomplete-the outflow surface in the full problem should be a free boundary on which three boundary conditions are imposed (rather than two, as in this paper). We anticipate that, when a code incorporating the free boundary is developed, flows deviating more substantially from the radial solution will be calculable.

Comparison with experiment is a long-term goal for simulations of the type reported here. Several extensions of this work are needed for such comparisons to be 


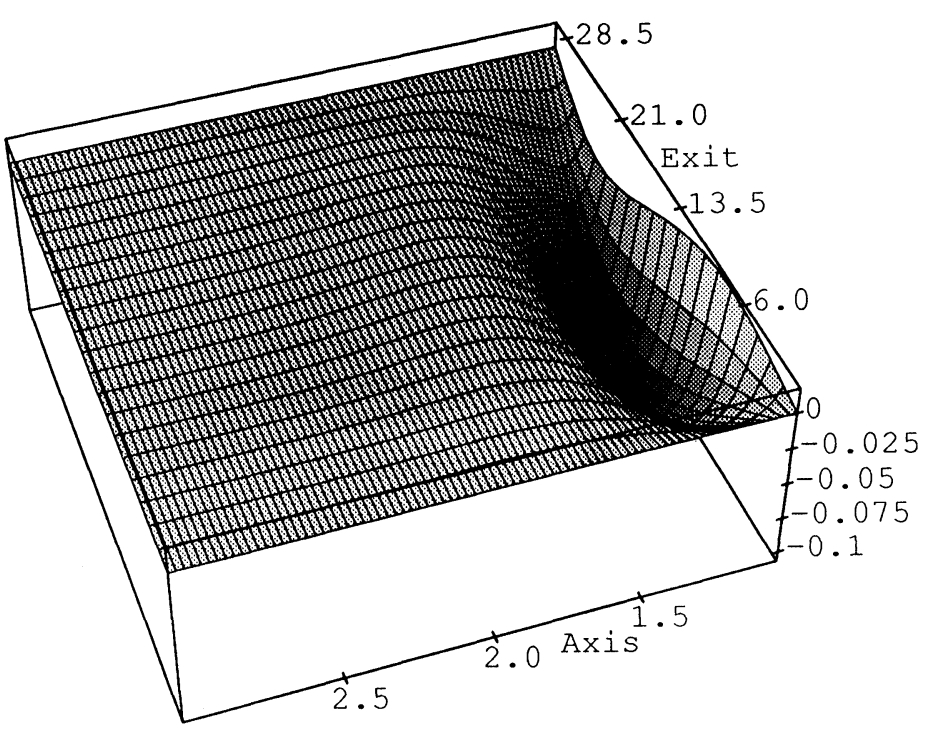

FIG. 16. $v_{\theta}\left(\theta_{w}=30^{\circ}\right.$, boundary condition (23b) with $\left.\varepsilon=0.4\right)$.

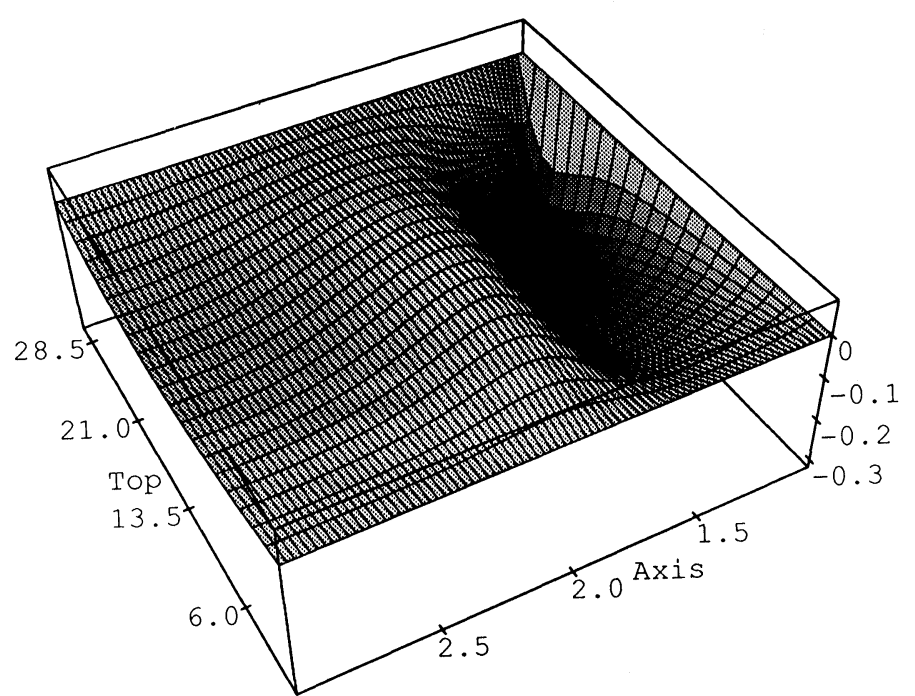

FIG. 17. $T_{r \theta}\left(\theta_{w}=30^{\circ}\right.$, boundary condition (23b) with $\left.\varepsilon=0.4\right)$.

meaningful. Most significantly, (i) equations that change from elliptic to hyperbolic must be handled to model the free boundaries between flowing and stagnant regions (shear bands), and (ii) time-dependent computations are needed to compute oscillatory phenomena.

\section{REFERENCES}

[1] R. E. BANK, D. J. Rose, AND W. FICHTNER, Numerical methods for semi-conductor device simulation, SIAM J. Sci. Statist. Comput., 4 (1983), pp. 416-435.

[2] G. Baxter, R. Behringer, T. FAGert, AND G. Johnson, Pattern formation in flowing sand, Phys. Rev. Lett., 62 (1989), pp. 2825-2826. 
[3] C. Coulomв, Essai sur une application des règles de maximis et minimis à quelques problèmes de statique, relatifs à l'architecture, Memoirs Mathematiques de l'Acadamie Royale des Sciences (Paris), 7 (1776), pp. 343-382.

[4] A. Jenike, Gravity flow of bulk solids, Bulletin No. 108, Utah Engineering Experimental Station, University of Utah, Salt Lake City, UT, 1961.

[5] D. G. SCHAEFFER, Instability in the evolution equations describing incompressible granular flow, J. Differential Equations, 66 (1987), pp. 19-50. 